\title{
ANALYTICAL DESIGN OF NONLINEAR CONTROL SYSTEMS
}

\author{
UDC 681.511.4:681.327.2
}

\author{
Anatoly Gaiduk ${ }^{1}$, Nadežda Stojković ${ }^{2}$, Elena Plaksienko \\ ${ }^{1}$ Southern Federal University, Department of Control Systems, Taganrog, Russia \\ ${ }^{2}$ University of Niš, Faculty of Electronic Engineering, Department of General Education \\ Republic of Serbia \\ ${ }^{3}$ Taganrog Institute of Economics and Management, Department of Mathematics and \\ Informatics, Taganrog, Russia
}

\begin{abstract}
This paper presents two approaches to analytical design of nonlinear control systems using transformation of plant equations into quasilinear forms or into Jordan controlled form. Settlement expressions of corresponding analytical methods of control systems design are obtained as results. These methods can be applied if the plant's nonlinear functions are differentiable, the plant is controllable and the additional conditions are satisfied. The suggested methods provide asymptotical stability of the equilibrium in a bounded domain of the state space or global stability and also desirable performance of transients. Examples of control systems design by the suggested analytical methods are given.
\end{abstract}

Key words: plant, nonlinearity, nonlinear transformation, quasilinear form, Jordan controlled form, controllability, control, design, system

\section{INTRODUCTION}

Control design problem for nonlinear plants, despite of an old history of development, still has no exhaustive solution. It has been considered in many works. However, the majority of the proposed methods have a bounded scope. Therefore, the new control design methods for nonlinear plants are actual. The transformation method of the plant equations to some simple form is widely used for solving linear and nonlinear control systems problem. This approach simplifies the solution of this problem and makes it analytical. In nonlinear cases the plant equations are transformed to the various forms. It can be normal canonical control form [1-4], triangular form [5, 6], Lukyanov-Utkin regular form [7], quasilinear form [8 - 10], Jordan controlled form (JCF) [11 - 13] and others. Transformation of the humanoid robot equations to a controllability canonical form has allowed designing a control system which compensates influence of external disturbances [2, 3] and parameters

Received April 16, 2016

Corresponding author: Anatoly R. Gaiduk

Southern Federal University, Department of Control Systems, Nekrasovskiy 44, 347928, Taganrog, Russia

E-mail: gaiduk_2003@mail.ru 
uncertainties [3]. If the plant equations are represented in the triangular form it is easy to apply the backstepping method to design stabilizing control or to design an adaptive control $[5,6]$. The Lukyanov-Utkin regular form of the plant equations allows decoupling the high dimension design problem on several tasks of the smaller dimensions [7].

The purpose of this paper is the representation of rather effective analytical approaches to the design of nonlinear control systems on the basis of transformation of the plants and systems equations to the quasilinear or Jordan controlled form. These approaches allow analytically finding controls, which provides stability of the system's equilibrium [9], desired duration of transients and full compensation of influence of bounded external disturbances [12, 13]. Mathematical basis of these approaches are differentiability plant nonlinearities, plant controllability and independence from the integration way of the curvilinear integral [14].

This article is organized as follows. The problem of nonlinear control systems design is given in chapter 2. Transformation of the nonlinear equations of dynamic plants and systems to the quasilinear form is presented in chapter 3. Features of this transformation and the suggested analytical method of nonlinear control systems design on the basis of this form are given in chapter 4. The approach to design problem of the nonlinear control systems on the basis of a Jordan controlled form (JCF) is considered in chapter 5. The analysis shows: the equations of many real nonlinear plants have JCF or may be represented in JCF by simple transformation. In the final chapter the corresponding examples of control systems design are resulted.

\section{STATEMENT OF CONTROL SySTEM DESIGN PROBLEM}

Assume some controlled plant is described by the equation

$$
\dot{x}=f\left(x, u_{0}\right),
$$

where $x \in R^{n}$ is a measured state vector; $f(x, u) \in R^{n}$ is a nonlinear differentiated vector-function; $u_{0}=u_{0}(x)$ is scalar control. Let be $x^{\circ}=x^{\circ}\left(t, u_{0}^{\circ}\right)$ is a vector that describes the unperturbed motion of the system (1); $u_{0}^{\circ}$ is appropriate control. Enter the deviations $\tilde{x}=x-x^{\circ}$ and $u=u_{0}-u_{0}^{\circ}$. The equations of the system (1) in deviations are recorded in a next form:

$$
\dot{\tilde{x}}=f(\tilde{x}, u),
$$

where $f(\tilde{x}, u)=\left[f\left(x^{\circ}+\tilde{x}, u_{0}^{\circ}+u\right)-f\left(x^{\circ}, u_{0}^{\circ}\right)\right]$ is a nonlinear differentiable vector-function.

Usually, when $u=0$ the equilibrium $\tilde{x} \equiv 0$ of the plant (2) is unstable or the processes in this plant are unsatisfactory. The control system design problem consists in the definition of the control $u=u(\tilde{x})$ so that equilibrium $\tilde{x} \equiv 0$ of the plant (2) was asymptotically stable, at least, in an bounded domain $\Omega$, i.e.

$$
\lim _{t \rightarrow \infty} \tilde{x}\left(t, \tilde{x}_{0}, u(\tilde{x})\right)=0, \tilde{x}_{0} \in \Omega_{0} \in \Omega \in R^{n}, \tilde{x} \in \Omega \in R^{n},
$$

where $\Omega_{0}$ is a bounded attraction domain of the equilibrium $\tilde{x} \equiv 0$. This control should provide also desired duration and character of transients. 
The method of the design problem solution depends, first of all, on properties of the nonlinear vector-function $f(\tilde{x}, u)$. In the beginning we shall consider as the quasilinear form of the equation (2) is applied to this purpose and then - the Jordan controlled form. Conditions on the nonlinear vector-function $f(\tilde{x}, u)$, at which the design problem has the solution, will be shown.

\section{CONTROL SYSTEM DESIGN USING QUASILINEAR FORM}

Let the nonlinear vector-function $f(\tilde{x}, u)$ in equation (2) such that

$$
f(0,0)=0, \frac{\partial f_{i}(\tilde{x}, u)}{\partial u}=f_{i u}(\tilde{x}), \tilde{x} \in \Omega \in R^{n}, \quad\|\tilde{x}\| \leq M_{\Omega}<\infty .
$$

where $M_{\Omega}$ is a number dependent on the sizes of domain $\Omega$; $\|\tilde{x}\|$ - Euclidean norm. Before passing to the solution of the statement problem, we shall define the term «quasilinear form» of nonlinear functions and nonlinear vector-functions [9].

Suppose some nonlinear function $f(x)=f\left(x_{1}, \ldots, x_{n}\right)$ of variables $x_{1}, \ldots, x_{n}$ is differentiable. Then it can be presented always as follows:

$$
f(x)=a^{T}(x) x+f(0)=\left[\begin{array}{lll}
a_{1}(x) & \ldots & a_{n}(x)
\end{array}\right] x+f(0),
$$

where $a^{T}(x)$ is some functional $n$-vector and $a_{i}(x)$ are its components depend on a way of integration of the partial derivatives $f_{i}(x)=\partial f(x) / \partial x_{i}$ from a point $x=0$ to a point $x$. Various ways of integration give various quasilinear representations of the nonlinear function [8, 9, 14]. We shall use the following expressions for definition of the components $a_{i}(x)$ :

$$
a_{i}^{I}(x)=\int_{0}^{1} f_{i}\left(x_{1}, \ldots x_{i-1}, x_{i} \theta, 0, \ldots 0\right) d \theta, i=\overline{1, n},
$$

where $\theta$ is a scalar integration variable.

The validity of the expressions (5), (6) will be shown on examples. Let us consider function $f_{*}(x)=x_{1} x_{2}^{2}+x_{2} x_{3}^{3}+x_{1}^{4} x_{3}+v$, where $x_{1}, x_{2}, x_{3}$ there are independent variables and $v$ is some constant. The function $f_{*}(x)$ is differentiable; therefore there are its partial derivatives:

$$
f_{* 1}(x)=x_{2}^{2}+4 x_{1}^{3} x_{3}, f_{* 2}(x)=2 x_{1} x_{2}+x_{3}^{3}, f_{* 3}(x)=3 x_{2} x_{3}^{2}+x_{1}^{4},
$$

and $f_{*}(0)=v$. Substituting the received expressions (7) for partial derivatives in the formula (6), we shall find: $a_{* 1}(x)=0, a_{* 2}(x)=x_{1} x_{2}, a_{* 3}(x)=x_{1}^{4}+x_{2} x_{3}^{2}$, i.e. the vector $a_{*}^{T}(x)=\left[\begin{array}{lll}0 & x_{1} x_{2} & x_{1}^{4}+x_{2} x_{3}^{2}\end{array}\right]$. The received vector and the formula (5) give the consider nonlinear function $f_{*}(x)$.

The expressions (5) and (6) are fair and in relation to differentiated vector-functions with replacement of a vector $a^{T}(x)$ by a corresponding functional matrix. Let, for example, $x^{T}=\left[\begin{array}{lll}x_{1} & x_{2} & x_{3}\end{array}\right]$ and $f_{* *}^{T}(x)=\left[\begin{array}{lll}3 x_{2}+4 x_{1}^{2} & 7 x_{2} x_{3}+2 \sin x_{1} & 1,2 x_{3} x_{1}+x_{3}^{3}\end{array}\right]$. The expressions (7), (6) applied to the components of this vector-function give a matrix 


$$
A(x)=\left[\begin{array}{ccc}
4 x_{1} & 3 & 0 \\
2 \omega\left(x_{1}\right) & 0 & 7 x_{2} \\
0 & 0 & 1,2 x_{1}+x_{3}^{2}
\end{array}\right],
$$

where $\omega\left(x_{1}\right)=\left(\sin x_{1}\right) / x_{1}$. The validity of the expression $f_{* *}(x)=A(x) x+f_{* *}(0)$ is evidently.

Though quasilinear representations of a differentiable function and a vector-function are not unique, but any quasilinear form describes the given nonlinearity precisely, in difference, for example, from «the first approximation» $[4,5]$.

The vector-function $f(\tilde{x}, u)$ from the equation (2) satisfies the conditions (4), therefore according to expressions (5), (6) this equation can be submitted as follows:

$$
\dot{\tilde{x}}=A(\tilde{x}) \tilde{x}+b(\tilde{x}) u, \tilde{x} \in \Omega \in R^{n}, \quad\|\tilde{x}\| \leq M_{\Omega}<\infty .
$$

Here $A(\tilde{x})=\left[a_{i j}(\tilde{x})\right]$ is a functional $n \times n$-matrix, $b(\tilde{x})=\left[b_{i}(\tilde{x})\right]$ is functional $n$-vector and $b_{i}(\tilde{x})=f_{i u}(\tilde{x})$. The equation (8) is the quasilinear form of the equation (2).

In summary, we shall emphasize: the right parts of the equations (2) and (8) are completely identical with all $\tilde{x} \in \Omega \in R^{n},\|\tilde{x}\| \leq M_{\Omega}<\infty$, i.e. the quasilinear form (8) is the exact representation of the nonlinear differential equations such as (2), satisfying conditions (4). The quasilinear form of nonlinear equations is close to linear; therefore the well developed analytical methods of the linear control theory can be applied to the problem solution of the nonlinear systems design.

The control at equation (8) is searched also in the quasilinear form:

$$
u(\tilde{x})=-k^{T}(\tilde{x}) \tilde{x}=-\sum_{i=1}^{n} k_{i}(\tilde{x}) \tilde{x}_{i}, \tilde{x} \in \Omega \in R^{n}, \quad\|\tilde{x}\| \leq M_{\Omega}<\infty .
$$

Here $k_{i}(\tilde{x})$ are some nonlinear functions. Next equation follows from the expressions (8) and (9):

$$
\dot{\tilde{x}}=D(\tilde{x}) \tilde{x},
$$

where

$$
D(\tilde{x})=A(\tilde{x})-b(\tilde{x}) k^{T}(\tilde{x}) .
$$

The characteristic polynomial of the functional matrix $D(x)$ (11), in view of the identity $\operatorname{det}\left(M+b k^{T}\right)=\operatorname{det} M+k^{T}(\operatorname{adj} M) b$ [9], it is possible to present as follows:

$$
D(p, \tilde{x})=\operatorname{det}(p E-D(\tilde{x}))=A(p, \tilde{x})+k^{T}(\tilde{x}) \operatorname{adj}(p E-A(\tilde{x})) b(\tilde{x})
$$

or

$$
D(p, \tilde{x})=A(p, \tilde{x})+\sum_{i=1}^{n} k_{i}(\tilde{x}) B_{i}(p, \tilde{x}) .
$$

Here adj is the adjunct matrix $[9,14]$ and polynomials are determined by next expressions

$$
A(p, \tilde{x})=\operatorname{det}(p E-A(\tilde{x}))=p^{n}+\sum_{i=0}^{n-1} \alpha_{i}(\tilde{x}) p^{i},
$$




$$
B_{i}(p, \tilde{x})=e_{i} \operatorname{adj}(p E-A(\tilde{x})) b(\tilde{x})=\sum_{j=0}^{n-1} \beta_{i j}(\tilde{x}) p^{j}, i=\overline{1, n},
$$

where $e_{1}=\left[\begin{array}{llll}1 & 0 & \ldots & 0\end{array}\right], e_{2}=\left[\begin{array}{llll}0 & 1 & \ldots & 0\end{array}\right], \ldots, e_{n}=\left[\begin{array}{llll}0 & 0 & \ldots & 1\end{array}\right]$.

Let, according to stability and necessary performance of the closed nonlinear system, the desirable characteristic polynomial of the matrix $D(\tilde{x})$ from equation (10) be appointed the following kind:

$$
D^{*}(p)=p^{n}+\delta_{n-1}^{*} p^{n-1}+\cdots+\delta_{1}^{*} p+\delta_{0}^{*} .
$$

The polynomial (15) satisfies the Gurvits criteria. If the polynomial (15) is to substitute in the equation (12) instead of the polynomial $D(p, x)$, a polynomial equation is formed which is equivalent in view of expressions (13), (14) to the following algebraic system:

$$
\left[\begin{array}{cccc}
\beta_{10} & \beta_{20} & \cdots & \beta_{n 0} \\
\beta_{11} & \beta_{21} & \cdots & \beta_{n 1} \\
\vdots & \vdots & \ddots & \vdots \\
\beta_{1 n-1} & \beta_{2 n-1} & \cdots & \beta_{n, n-1}
\end{array}\right]\left[\begin{array}{c}
k_{1} \\
k_{2} \\
\vdots \\
k_{n}
\end{array}\right]=\left[\begin{array}{c}
\eta_{0} \\
\eta_{1} \\
\vdots \\
\eta_{n-1}
\end{array}\right] .
$$

Here $\eta_{i}=\eta_{i}(\tilde{x})$ are the coefficients of the polynomials difference: $D^{*}(p)-D(p, \tilde{x})=$ $\eta_{0}(\tilde{x})+\eta_{1}(\tilde{x}) p+\ldots+\eta_{n-1}(\tilde{x}) p^{n-1}$. In the system (16) the arguments of the functions are lowered for brevity.

Solution of the algebraic system (16) defines the functions $k_{i}(\tilde{x})$ from control (9) of the closed system (2), (9) or the system (10). The algebraic system (16) has the solution if the next condition satisfies:

$$
\operatorname{det} U(\tilde{x})=\operatorname{det}\left[b(\tilde{x}) A(\tilde{x}) b(\tilde{x}) \ldots A^{n-1}(\tilde{x}) b(\tilde{x})\right] \neq 0, \tilde{x} \in \Omega \in R^{n}, \quad\|\tilde{x}\| \leq M_{\Omega}<\infty .
$$

Note, the condition (17) is the controllability condition of the nonlinear plant (8) [9, 10]. If the matrix $A$ and the vector $b$ in the equation (8) are constants then the inequality (17) passes in the well known Kalman controllability condition.

Thus, if the vector $k(x)(9)$ is determined by the expressions (13) - (16) the constant matrix $D(0)$ and the equilibrium $x \equiv 0$ of the nonlinear closed system (10) are stable. The majority of the control systems designed by this method are asymptotically stable in the bounded domain $\Omega \in R^{n}$. Only in some cases the equilibrium $x \equiv 0$ of this system is globally asymptotically stable [9]. Hence, if the plant equations are transformed to the quasilinear form, then expressions (9) - (16) allow finding control by which the condition (3) is carried out. Bounded attraction domain $\Omega_{0}$ from the condition (3) can be found using Lyapunov's function, constructed for stable system $\dot{\tilde{x}}=D(0) \tilde{x}$ or by computer simulation of the close system (10). It is easy to see, that expressions (13) - (16) can be applied to design of a modal control for linear plants with constant parameters. But in this case they provide the global stability of the closed system. This approach gives possibilities to design of the control systems which the equilibrium $x \equiv 0$ are globally stable. Corresponding method is given at [15].

The application of the quasilinear form and the expressions (9) - (16) to control systems design shall be shown on an example below 


\section{CONTROL System DESIGN USING JCF OF PLANT EQUATIONS}

Suppose the equations (2) of the plant (1) in the scalar form look like:

$$
\begin{gathered}
\dot{\tilde{x}}_{i}=\phi_{i}\left(\tilde{x}_{1}, \ldots, \tilde{x}_{i+1}\right), i=\overline{1, n-1}, \\
\dot{\tilde{x}}_{n}=\phi_{n}\left(\tilde{x}_{1}, \ldots, \tilde{x}_{n}\right)+u,
\end{gathered}
$$

where $\phi_{i}\left(\tilde{x}_{1}, \ldots, \tilde{x}_{i+1}\right)=f_{i}\left(\bar{x}_{i+1}^{\circ}+\overline{\tilde{x}}_{i+1}\right)-f_{i}\left(\bar{x}_{i+1}^{\circ}\right)=\phi_{i}\left(\overline{\tilde{x}}_{i+1}\right), i=\overline{1, n-1}$ and $f_{n}\left(x+\tilde{x}, u_{0}^{\circ}+u\right)-f_{n}\left(x^{\circ}, u_{0}^{\circ}\right)$ $=\phi_{n}(\tilde{x})+u$ are differentiable $n-i$ time nonlinear function; $\overline{\tilde{x}}_{i}=\left[\tilde{x}_{1} \ldots \tilde{x}_{i}\right]^{T}$ is a subvector and $\overline{\tilde{x}}_{n}=\tilde{x}$ evidently; $u=u(\tilde{x})$ is the search control. The controllability conditions of the system (18), (19) can be written down as follows:

$$
\left|\frac{\partial \phi_{i}\left(\tilde{x}_{1}, \ldots, \tilde{x}_{i+1}\right)}{\partial \tilde{x}_{i+1}}\right| \geq \varepsilon \neq 0, i=\overline{1, n-1}, \tilde{x} \in \Omega \in R^{n},
$$

where $\varepsilon$ there is any positive number. The domain $\Omega$ includes the equilibrium $\tilde{x}=0$.

Definition. If the system of equations (18), (19) satisfies the conditions (20), it is called "Jordan controlled form" [9, 11].

Evidently, the Jordan controlled form is a generalization of the known triangular form of the equations of nonlinear plants [5,6].

To solve the control system design problem, in the first, the transformation of the state vector $\tilde{x}$ of the system (18) - (20) to new state vector $w$ is determined as follows

where

$$
w=w(\tilde{x})=\left[\begin{array}{llll}
w_{1}(\tilde{x}) & w_{2}(\tilde{x}) & \ldots & w_{n}(\tilde{x})
\end{array}\right]^{T},
$$

$$
w_{1}=\tilde{x}_{1}, w_{i}\left(\overline{\tilde{x}}_{i}\right)=\sum_{v=1}^{i-1} \frac{\partial w_{i-1}}{\partial \tilde{x}_{v}} \phi_{v}\left(\overline{\tilde{x}}_{v+1}\right)+\lambda_{i-1} w_{i-1}\left(\overline{\tilde{x}}_{i-1}\right), i=\overline{2, n},
$$

and $\lambda_{i}$ are some constants. The transformation $w(\tilde{x})(21),(22)$ is bounded and is convertible by virtue of conditions (20), i.e. in the domain $\Omega \in R^{n}$ there is a bounded inverse transformation $\tilde{x}=\tilde{x}(w)$ such that $\tilde{x}(w)=\tilde{x}(w(\tilde{x}))=\tilde{x}$.

The stabilizing control $u=u(\tilde{x})$ for plant (18), (19) is determined by the expressions

$$
\begin{gathered}
u(\tilde{x})=-\gamma_{1}^{-1}(\tilde{x})\left[\gamma_{2}(\tilde{x})+\lambda_{n} w_{n}(\tilde{x})\right]-\phi_{n}(\tilde{x}), \tilde{x} \in \Omega, \\
\gamma_{1}(\tilde{x})=\frac{\partial w_{n}(\tilde{x})}{\partial \tilde{x}_{n}}=\prod_{i=1}^{n-1} \frac{\partial \phi_{i}\left(\overline{\tilde{x}}_{i+1}\right)}{\partial \tilde{x}_{i+1}}, \gamma_{2}(\tilde{x})=\sum_{v=1}^{n-1} \frac{\partial w_{n}(\tilde{x})}{\partial \tilde{x}_{v}} \tilde{\phi}_{v}\left(\overline{\tilde{x}}_{v+1}\right), \tilde{x} \in \Omega,
\end{gathered}
$$

where $\lambda_{n} \geq \varepsilon>0$; variables $w_{n}(\tilde{x})$ are determined by the expressions (22) with $\lambda_{i} \geq \varepsilon>0$, $i=\overline{1, n-1}[11-13]$.

The close system (18) - (20) with control (22) - (24) is described in the new variables $w_{i}=w_{i}\left(\overline{\tilde{x}}_{i}\right), i=\overline{1, n}$ by the expressions 


$$
\dot{w}=\Lambda_{n} w, \Lambda_{n}=\left[\begin{array}{cccc}
-\lambda_{1} & 1 & \ldots & 0 \\
0 & -\lambda_{2} & \ddots & 0 \\
\vdots & \vdots & \ddots & 1 \\
0 & 0 & \ldots & -\lambda_{n}
\end{array}\right]
$$

Note, the condition (20) ensures the existence of the stabilizing control (23) in the domain $\Omega \in R^{n}$. The matrix $\Lambda_{n}$ (25) coincides with the $n \times n$ cell Jordan [16, p. 142] if $\lambda_{i}=-\lambda$, $i=\overline{1, n}$. Just therefore the system of equations (18), (19) is called "Jordan controlled form", if condition (20) is carried out in some domain $\Omega \in R^{n}$. Evidently, the system (25) is asymptotically stable if $\lambda_{i} \geq \varepsilon>0, i=\overline{1, n}$. Since the transformation (21) - (22) is convertible and bounded, then the equilibrium $\tilde{x}=0$ of the system (18), (19), (22) - (24) with $\lambda_{i} \geq \varepsilon>0, i=\overline{1, n}$ also asymptotically stable in the domain $\Omega \in R^{n}$.

So, if the nonlinear plant equation (2) is represented in the JCF (18), (19) and conditions (20) are carried out, then the expressions (21) - (24) give the analytical design method of the nonlinear control systems. The transformation method of the nonlinear equations (1) general view to JCF is not known. However in practice very much many plants can be transformed to JCF by change of indexes of their variables. In some cases the methods suggested in $[6,7]$ can be applied for transformation of the equations (1) to JCF.

Transformation of the nonlinear plant equations to this form allows to design by the analytical way not only the stabilizing controls such as (23), but also the control optimal in sense of uncertain nonlinear-quadratic criteria [17].

\section{EXAMPLES}

Example 1. The plant is described in deviations by the equations:

$$
\dot{\tilde{x}}_{1}=\tilde{x}_{2}, \dot{\tilde{x}}_{2}=-a_{21} \sin \tilde{x}_{1}+a_{23} \tilde{x}_{3}, \dot{\tilde{x}}_{3}=-\varphi_{2}\left(\tilde{x}_{2}\right)-\varphi_{3}\left(\tilde{x}_{3}\right)+u \text {. }
$$

where $a_{21}, a_{23} \neq 0$ are numbers, $\varphi_{2}\left(\tilde{x}_{2}\right), \varphi_{3}\left(\tilde{x}_{3}\right)$ are differentiable functions. The state variables $\tilde{x}_{1}, \tilde{x}_{2}, \tilde{x}_{3}$ are measured. To find the control $u=-k(\tilde{x}) \tilde{x}$ by which the equilibrium point $\tilde{x} \equiv 0$ of the plant (26) will be asymptotically stable.

In the equations (26) the vector-function $f(0,0)=0$, therefore according to the formula (6) the quasilinear equation (8) corresponds to the equations (26) with

$$
A(\tilde{x})=\left[\begin{array}{ccc}
0 & 1 & 0 \\
\omega\left(\tilde{x}_{1}\right) & 0 & a_{23} \\
0 & -a_{32}\left(\tilde{x}_{2}\right) & -a_{33}\left(\tilde{x}_{3}\right)
\end{array}\right], b(x)=\left[\begin{array}{l}
0 \\
0 \\
1
\end{array}\right],
$$

where $\omega\left(\tilde{x}_{1}\right)=-a_{21} \tilde{x}_{1}^{-1} \sin \tilde{x}, a_{3 j}\left(\tilde{x}_{j}\right)=\tilde{x}_{j}^{-1} \varphi_{j}\left(\tilde{x}_{j}\right), j=2,3$.

It is easy to see that the equation (8) in view of the expressions (27) is the exact representation of the equations of the nonlinear plant (26). In this case $\operatorname{det} U(x)=-a_{23}^{2} \neq 0$, the condition (17) is carried out, i.e. the solution of the design problem exists.

Passing to its definition, we find by the formulas (13) and (14) the polynomials: $A(p, \tilde{x})=p^{3}+a_{33}\left(\tilde{x}_{3}\right) p^{2}+\alpha_{1}(\tilde{x}) p+\alpha_{0}(\tilde{x}), \quad B_{1}(p, \tilde{x})=a_{23}, \quad B_{2}(p, \tilde{x})=a_{23} p, \quad B_{3}(p, \tilde{x})=p^{2}-\omega\left(\tilde{x}_{1}\right)$, where $\alpha_{1}(\tilde{x})=a_{23} a_{32}\left(\tilde{x}_{2}\right)-\omega\left(\tilde{x}_{1}\right), \alpha_{0}(\tilde{x})=-a_{33}\left(\tilde{x}_{3}\right) \omega\left(\tilde{x}_{1}\right)$. The desirable polynomial $D^{*}(p)=$ $p^{3}+\delta_{2}^{*} p^{2}+\delta_{1}^{*} p+\delta_{0}^{*}$ satisfies to the Gurvits criterion and the system (17) looks like here: 


$$
\left[\begin{array}{ccc}
a_{23} & 0 & -\omega\left(\tilde{x}_{1}\right) \\
0 & a_{23} & 0 \\
0 & 0 & 1
\end{array}\right]\left[\begin{array}{l}
k_{1} \\
k_{2} \\
k_{3}
\end{array}\right]=\left[\begin{array}{c}
\delta_{0}^{*}+a_{33}\left(\tilde{x}_{3}\right) \omega\left(\tilde{x}_{1}\right) \\
\delta_{1}^{*}-a_{23} a_{32}\left(\tilde{x}_{2}\right)+\omega\left(x_{1}\right) \\
\delta_{2}^{*}-a_{33}\left(\tilde{x}_{3}\right)
\end{array}\right] .
$$

The solution of this system are functions: $k_{3}(\tilde{x})=\delta_{2}^{*}-a_{33}\left(\tilde{x}_{3}\right), k_{2}(\tilde{x})=a_{23}^{-1}\left(\delta_{1}^{*}+\omega\left(\tilde{x}_{1}\right)\right)$ $-a_{32}\left(\tilde{x}_{2}\right), k_{1}(\tilde{x})=a_{23}^{-1}\left(\delta_{0}^{*}+\delta_{2}^{*} \omega\left(\tilde{x}_{1}\right)\right)$. In according to the expression (9) this solution leads to control:

$$
u(\tilde{x})=-a_{23}^{-1} \delta_{0}^{*} \tilde{x}_{1}+\delta_{2}^{*} \sin \tilde{x}_{1}-a_{23}^{-1}\left(\delta_{1}^{*}+\omega\left(\tilde{x}_{1}\right)\right) \tilde{x}_{2}+\varphi_{2}\left(\tilde{x}_{2}\right)-\delta_{2}^{*} \tilde{x}_{3}+\varphi_{3}\left(\tilde{x}_{3}\right)
$$

It is easy to establish the characteristic polynomial $D(p, x)$ calculated under the expression (11) is equal to the desirable polynomial $D^{*}(p)$. Hence, the equilibrium point $x \equiv 0$ of the closed system (26), (28) is asymptotically stable in some bounded domain [9].

The closed system was simulated in MATLAB with $\varphi_{2}\left(\tilde{x}_{2}\right)=3 \operatorname{arctg}\left(\tilde{x}_{2}\right), \varphi_{3}\left(\tilde{x}_{3}\right)=$ $9 \operatorname{arctg}\left(\tilde{x}_{3}\right) ; a_{21}=2, a_{23}=2.5 ; \delta_{0}^{*}=81, \delta_{1}^{*}=27, \delta_{2}^{*}=9, \delta_{3}^{*}=1$ and $\tilde{x}_{0}=\left[\begin{array}{lll}0.25 & 3 & 0\end{array}\right]$. Schedules of the variables of the designed system (26), (28) are shown in Fig. 1. These schedules allow concluding, that the designed system is asymptotically stable and duration of the transients does not exceed 4.0 second. The schedule of the control signal is shown in Fig. 2. Apparently, the control signal is bounded during all transient.

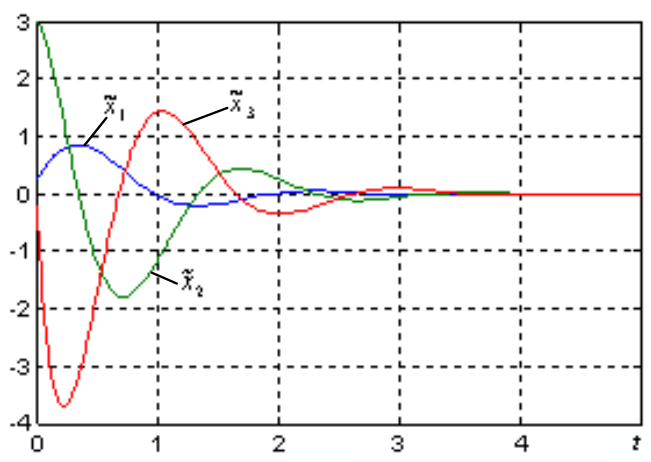

Fig. 1 Schedules of the variables

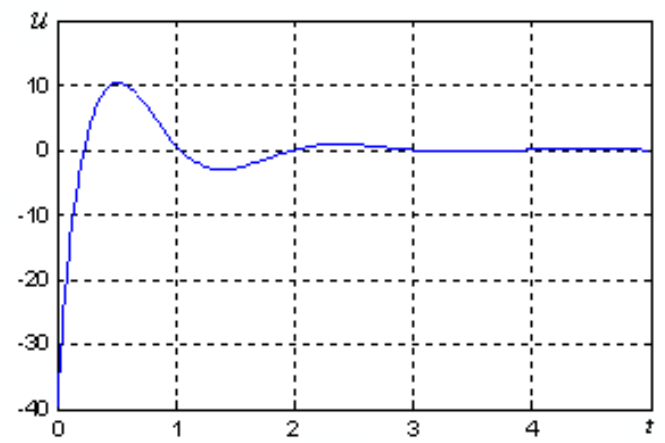

Fig. 2 Schedule of the control $u$ 
Example 2. Suppose a nonlinear plant is described by the equations

$$
\dot{x}_{1}=x_{2} x_{3}+u, \dot{x}_{2}=x_{3}, \dot{x}_{3}=\left(1+x_{2}^{2}\right) x_{1} .
$$

Using the design method with application JCF to find the control $u(\tilde{x})$ by which the equilibrium point $x \equiv 0$ of the plant (29) will be asymptotically stable and time response not more then 3.5 second.

The form of the equations (29), evidently, does not meet to the form of the equations (18), (19). That the equations (29) had this form, we shall designate the state variable so: $x_{1}=\tilde{x}_{3}, x_{2}=\tilde{x}_{1}, x_{3}=\tilde{x}_{2}$. The resulting equations of the plant look like:

$$
\dot{\tilde{x}}_{1}=\tilde{x}_{2}=\phi_{1}(\tilde{x}) ; \dot{\tilde{x}}_{2}=\left(1+\tilde{x}_{1}^{2}\right) \tilde{x}_{3}=\phi_{2}(\tilde{x}) ; \dot{\tilde{x}}_{3}=\tilde{x}_{1} \tilde{x}_{2}+u \text {. }
$$

Equations (30) satisfy the conditions (20), since $\partial \phi_{1}(\tilde{x}) / \partial \tilde{x}_{2}=1$ and $\partial \phi_{2}(\tilde{x}) / \partial \tilde{x}_{3}=\left(1+\tilde{x}_{1}^{2}\right) \geq 1$ for all $\tilde{x} \in R^{3}$. Therefore, equations (30) have JCF, and the design task has a solution.

According to the proposed JCF method the transformation (21) is determined by the expressions (22), (30) and looks like:

$$
w_{1}=\tilde{x}_{1}, w_{2}=\tilde{x}_{2}+\lambda_{1} \tilde{x}_{1}, w_{3}=\left(1+\tilde{x}_{1}^{2}\right) \tilde{x}_{3}+\left(\lambda_{1}+\lambda_{2}\right) \tilde{x}_{2}+\lambda_{1} \lambda_{2} \tilde{x}_{1} .
$$

The transformation (31) is not singular, convertible and bounded for all $\tilde{x} \in R^{3},\|\tilde{x}\|<\infty$. The functions $\gamma_{1}(\tilde{x})$ and $\gamma_{2}(\tilde{x})$ are determined by the expressions (24), (31) and (30) as:

$$
\gamma_{1}(\tilde{x})=\left(1+\tilde{x}_{1}^{2}\right), \gamma_{2}(\tilde{x})=\left(2 \tilde{x}_{1} \tilde{x}_{3}+\lambda_{1} \lambda_{2}\right) \tilde{x}_{2}+\left(\lambda_{1}+\lambda_{2}\right)\left(1+\tilde{x}_{1}^{2}\right) \tilde{x}_{3} .
$$

Now the search control is written by the expression (23) as:

$$
u(\tilde{x})=-\tilde{x}_{1} \tilde{x}_{2}-\left(\gamma_{2}(\tilde{x})+\lambda_{3} w_{3}(\tilde{x})\right) /\left(1+\tilde{x}_{1}^{2}\right) .
$$

The expressions for this control in initial designations of the plant variables are obvious and here are not given. Transients of the nonlinear system (30), with controls (33), (31), (32) are submitted on Fig. 3. These schedules are received by simulation of the designed system in MATLAB with $\lambda_{1}=3, \lambda_{2}=5, \lambda_{1}=7$ and $x_{0}=\left[\begin{array}{lll}0.2 & 0 & 3\end{array}\right]^{T}$.

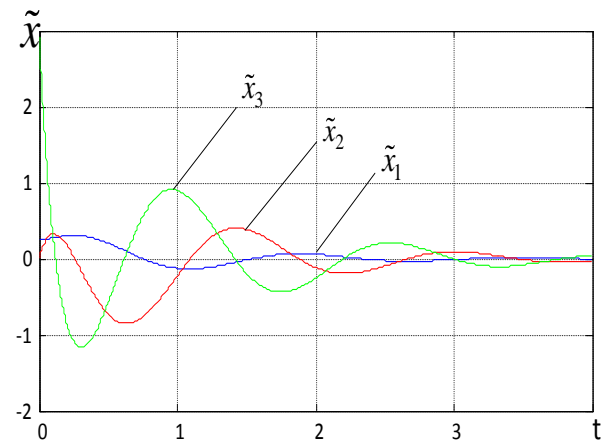

Fig. 3 Schedules of the nonlinear system transients 
Apparently, transient's character of the nonlinear control systems can be changed by a choice of the values of the control coefficients $\lambda_{i}, i=\overline{1, n}$.

Example 3. Nonlinear plant is described by the equations

$$
\dot{x}_{1}=\sin 0,1 x_{1}+b_{1} u, \dot{x}_{2}=u \sqrt{0,25 a^{2}+x_{2}},
$$

where $b_{1}$ and $a>0$ are constant parameters. To find the control $u(\tilde{x})$ by which the equilibrium $\tilde{x} \equiv 0$ of the plant (34) will be asymptotically stable.

The form of the equations (34) does not meet to the form of the equations (18), (19). A second order system $\dot{x}=f(x)+b(x) u$ can be transformed to JCF [9], if the following condition is carried out:

$$
K(x)=\left[G_{x 1}(x) \quad G_{x 2}(x)\right] b(x)-\left(b_{1 x}(x)+b_{2 x}(x)\right) G(x) \neq 0,
$$

where $G(x)=\operatorname{det}[f(x) b(x)], G_{x i}(x)=\partial G(x) / \partial x_{i}, b_{i x}(x)=\partial b_{i}(x) / \partial x_{i}, i=1,2$. In this case the functions $G(x)=b_{2}\left(x_{2}\right) \sin 0,1 x_{1}, K(x)=b_{1} b_{2}\left(x_{2}\right) \cos 0,1 x_{1}$ and $b_{2}\left(x_{2}\right)=\sqrt{0,25 a^{2}+x_{2}}$. Therefore, according to a condition (35), the equations (34) can be transformed to JCF until $\left|x_{1}\right|<5 \pi$ and $-0,25 a^{2}<x_{2} \leq M<\infty$. Transformation $x_{1}=\tilde{x}_{1}+b_{1} \tilde{x}_{2}, x_{2}=0,25\left[\left(a+\tilde{x}_{2}\right)^{2}-a^{2}\right]$ results the equations (34) in a kind: $\dot{\tilde{x}}_{1}=\sin 0,1\left(\tilde{x}_{1}+b_{1} \tilde{x}_{2}\right), \dot{\tilde{x}}_{2}=u$. These equations have JCF and, using expressions (22) - (26), we find control

$$
u(\tilde{x})=-\frac{\sin \beta(\tilde{x})}{b_{1}}-\frac{\left(\lambda_{1}+\lambda_{1}\right) \sin \beta(\tilde{x})+\lambda_{1} \lambda_{1} \tilde{x}_{1}}{0,1 b_{1} \cos \beta(\tilde{x})},\left|\tilde{x}_{1}+b_{1} \tilde{x}_{2}\right|<5 \pi,
$$

where $\beta(\tilde{x})=0,1\left(\tilde{x}_{1}+b_{1} \tilde{x}_{2}\right)$. The attraction domain of the equilibrium is bounded in this case. This domain can be determined by use of the linear character of the control systems (25) in variables $w_{i}, i=\overline{1, n}$ [9].

\section{CONCLUSION}

Representation of the equations of nonlinear plants in the quasilinear form or in the Jordan controlled form allows to find analytically the controls as nonlinear feedback on the state variables. These controls can provide asymptotic stability of the system equilibrium in some domain of the state space, duration and character of transients and also selective invariancy (astatic) to the bounded external disturbances or the control optimal in sense of uncertain nonlinear-quadratic criteria. Conditions of transformation possibility of the plant equations to the quasilinear form are very simple: nonlinear functions should be differentiable. Conditions of transformation to Jordan controlled form are more complex. Generally, these conditions are not found. However, the representation of the plant equations in the Jordan controlled form is not of a rigid restriction kind because the equations of the many real plants have this form or can be transformed to this form by replacement of the state variables.

Acknowledgement: The paper is a part of the running in SFU research is supported by the RFBR projects № 16-08-00013, № 16-58-00226 Bel. 


\section{REFERENCES}

[1] A. Isidori, Nonlinear control systems (2 ${ }^{\text {nd }}$ edition), New York: Springer-Verlag, 1989.

[2] V.O. Nikiforov, Nonlinear control system with rejection of the external determined disturbances. Izvestiya RAN. The theory and control systems, 4, 69-73, 1997.

[3] S. Savić, M. Raković, M. Penčić \& B. Borovac, Nonlinear motion of humanoid robot upper-body for manipulation task, Facta Universitatis. Series: Automatic Control and Robotics, vol. 13, 1, 1-14, 2014.

[4] D.P. Kim, Theory of automatic control. Vol. 2. Multivariable, nonlinear, optimal and adaptive systems. Moscow: Phizmatlit, 2004

[5] K.J. Åström \& B. Wittenmark, Adaptive control. New York: Addison-Wesley Publishing Company, 1995.

[6] M. Krstić, I. Kanellakopoulos \& P.V. Kokotović, Nonlinear and adaptive control design New York: John Willey and Sons, 1995.

[7] A.G. Lukyanov \& V.I. Utkin, Methods of transform of dynamic systems equations to regular form, Automation and Remote Control, 4, 5-13, 1981

[8] I.G. Egorov. To stability at whole of the zero solution of two differential equations system, Differential equations, vol. 27, no. 9, pp. 1554-1549, 1991

[9] A.R. Gaiduk, Theory and methods of automatic control systems analytical design, Moscow, Phizmatlit, 2012.

[10] V.A. Podchukaev, Analytical methods of the automatic control theory, Moscow, Phizmatlit, 2002.

[11] A.R. Gaiduk, Design of nonlinear systems based on the Jordan controlled form. Automation and Remote Control, 67(7), 1017-1027, 2006.

[12] A.R. Gaiduk, Control systems design with disturbance rejection based on JCF of the nonlinear plant equations. Facta Universitatis, Series: Automatic Control and Robotics. vol. 11, no. 2, pp. 81-90, 2012. [Online]. Available: facta.junis.ni.ac.rs/acar/acar201202/acar20120201.

[13] A.R. Gaiduk, Astatic control design for nonlinear plants on base of JCF. Transaction on Electrical and Electronic Circuits and Systems. vol 3, No. 2, pp. 80-84, 2013

[14] M. Fikhtengolts, Differential and integral calculus. Vol. 3, Moscow, Nauka, 1969.

[15] A.R. Gaiduk, N.M. Stojković, Analytical design of quasilinear control systems, FACTA UNIVERSITATIS. Series: Automatic Control and Robotics, vol. 13, no 2, pp. 73-84, 2014.

[16] P. Lankaster, Theory of matrices. New York: Academic Press, 1969.

[17] A.R. Gaiduk, E.A. Plaksienko, I.O. Shapovalov, "Optimal control based on Jordan controlled form", in Proceedings book of 14th International Conference on Circuits, Systems, Electronics, Control \& Signal Processing, CSECS '15, Selcuk University, Konya, Turkey, pp. 13-18, 2015. 